\title{
Investigación
}

\section{Filosofía y Derecho: Una experiencia académica en la Universidad de Ibagué}

\section{Philosophy and Law. An academic experience at Universidad de Ibagué}

Juan Manuel Barrero Arbeláez ${ }^{*}$

Recepción: 18/05/2020 • Aprobación: 10/07/2020 • Publicación: 30/10/2020

Para citar este artículo

Barrero Arbeláez, J. M. (2020). Filosofía y Derecho: Una experiencia académica en la Universidad de Ibagué. Dos mil tres mil, 22, e236.

https://doi.org/10.35707/dostresmil/22236

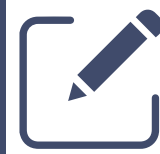

\section{(c) (1) $(9)$ \\ BY NC ND}

"Semillero de Investigación IUS SOFOS, Facultad de Derecho y Ciencias Políticas, Universidad de Ibagué, Colombia. ORCID: 0000-0002-6905-3763. Correo electrónico: juan.barrero@unibague.edu.co 
Resumen. El artículo presenta una reflexión del porqué resulta importante incluir en el plan de estudios del pregrado en derecho temas de filosofía política, ética, lógica y retórica, que son por tradición de interés de la filosofía. Además, se expone cómo puede aportar a un programa de derecho una relación académica continua con un programa o área de filosofía, todo esto a partir de la experiencia vivida en la Universidad de Ibagué.

Abstract. This article presents a reflection on why it is important to include political philosophy, ethics, logic and rhetoric topics in the undergraduate law curriculum, which are traditionally of interest to philosophy. In addition, it is set out how a law program can contribute to a continuous academic relationship with a program or area of philosophy, all this from the experience lived at Universidad de Ibagué.

Palabras claves

Educación jurídica, educación filosófica, formación ética, ética del abogado.

Key words

Legal education, philosophical education, ethical training, ethics of the lawyer. 


\section{Introducción}

El objetivo de este escrito es abordar cómo la filosofía en general, y un área de esta en particular, aporta a un programa de derecho y, en consecuencia, cómo contribuye a una mejor formación de los futuros abogados. Es importante destacar que no se tratan todas las posibles relaciones entre el derecho y la filosofía, sino los aportes que se generan en un programa de derecho al involucrar de manera significativa la filosofía en el proceso de formación de los estudiantes, a través de reformas al plan de estudios y de un trabajo conjunto con el área de filosofía. Esto se realizará a través de la experiencia vivida en la Universidad de Ibagué en años recientes y de la reflexión del autor.

Se considera que el estudio de temas filosóficos pertenecientes a la filosofía política, la éti$\mathrm{ca}$, la lógica y la retórica, resultan ser fundamentales para la formación de los futuros abogados en el desarrollo de competencias que les permitan ejercer la profesión de manera más idónea. Aunado a lo anterior, se sostiene que consolidar relaciones fuertes entre las áreas de derecho y filosofía dentro de una universidad, puede resultar provechoso para las partes involucradas, entre las que se encuentra la misma institución de educación superior.

Este escrito se desarrollará de la siguiente forma: primero se mostrará cómo se han involucrado la filosofía y el derecho en el plan de estudios del Programa de Derecho de la Universidad de Ibagué, y además se relata cómo ha sido la dinámica de trabajo conjunto adelantado entre el Programa de Derecho y el Área de Filosofía a través de las experiencias vividas en años recientes. Luego se presenta la opinión del autor sobre la relevancia de dichos cambios en el plan de estudios y cómo la relación con un área o programa de filosofía puede aportar a un programa de derecho. Por último, se formulan algunas conclusiones.

\section{La experiencia}

El Programa de Derecho de la Universidad de Ibagué hace parte de la Facultad de Derecho y Ciencias Políticas, y desde su creación, como parece ser la tradición en estos programas, ha vinculado asignaturas relacionadas con la filosofía, entre ellas la más representativa siempre ha sido Filosofía del Derecho; no obstante, además de esta, que es de suma relevancia, hay otras asignaturas con contenido filosófico, como lo son Teoría del Estado o Derecho Constitucional.

Así las cosas, la primera muestra de la experiencia referida es precisamente en relación con los cambios en el plan de estudios del Programa de Derecho de la Universidad de Ibagué. El anterior plan de estudios de 2004B contenía algunas asignaturas relacionadas con filosofía, como: Introducción al Derecho, Introducción al Derecho Público, Teoría del Estado y Ciencia Política, Filosofía del Derecho y Teoría Jurídica, Derecho Constitucional Colombiano, Derecho Político Comparado o Electividad VII, en la cual se podía optar por Teorías Políticas y Democracias Contemporáneas junto con ocho analíticas. Estas últimas son un conjunto de asignaturas cuya finalidad esencial era relacionar las asignaturas de cada uno de los módulos (distribuidos por áreas del derecho: público, privado, laboral, etc.), a partir de textos literarios, casos, y en general 
de cualquier reflexión que permitiera hacer un ejercicio de integración de conceptos, interpretación y argumentación.

En el pénsum 2016B se pueden observar varios cambios. Del plan anterior, se mantienen algunas de las asignaturas relacionadas con filosofía, pero se incluyen otras en las que pueden estudiarse temas de filosofía. Así, el nuevo plan de estudios contiene entre las asignaturas del pénsum antiguo y las nuevas, las siguientes: Introducción al Derecho, Ética y Política, Historia de las Ideas Políticas, cuatro electivas socio humanísticas, Lógica y Retórica, Teoría Constitucional y del Estado, Argumentación Jurídica, Constitucional Colombiano, Filosofía del Derecho y Ética Profesional.

Es importante destacar que, pese a que se retiraron las asignaturas analíticas, surgen otras que las reemplazan, algunas de ellas con alto contenido filosófico como Lógica y Retórica y Argumentación Jurídica, y otras que no han sido mencionadas pero que deberían abordar cuestiones filosóficas, sobre todo de filosofía política y ética. Dichas asignaturas son: Economía y Derecho, Uso Responsable de los Recursos Naturales y Globalización y Derecho. Aunado a lo anterior, muchos de los temas de estas nuevas asignaturas fueron trabajados en algunas analíticas, pero en las asignaturas nuevas necesariamente se deben estudiar.

En relación con los vínculos recientes entre el Programa de Derecho y el Área de Filosofía, estos se empezaron a consolidar con el trabajo desarrollado para la creación de la asignatura de Ética y Política. Esta se incluyó en los nuevos planes de estudio de los programas de la Universidad, y fue producto de un trabajo conjunto de profesores de filosofía, humanidades, ciencia política y derecho. En su creación, su contenido comprendía temas propios de filosofía, especialmente de ética, y temas de derecho y de ciencia política, y puede ser impartida por profesionales de distintas áreas del conocimiento, antropólogos, filósofos, abogados, politólogos, entre otros.

Las reuniones para la creación de esta asignatura propiciaron el diálogo entre las distintas áreas involucradas, lo cual resultó ser fundamental para sugerir los temas que debían tratarse en las clases. Pero además, entre otros aspectos, contribuyó para consolidar las relaciones entre el Área de Filosofía y el Programa de Derecho, que permitieron que se presentara el proyecto de investigación titulado: "Justicia. Un debate en torno a las cuestiones éticas contemporáneas" (2016-2017), que fue producto de la colaboración de profesores del Área de Filosofía pertenecientes al grupo de investigación Eulogos, con profesores del Programa de Derecho vinculados al grupo de investigación Zoon Politikon.

Este proyecto generó múltiples resultados, entre ellos algunos artículos de investigación sobre filosofía y derecho escritos por los profesores vinculados y además el Primer y el Segundo Encuentro de Filosofía Política y del Derecho (2017-2019). Eventos que contaron con la participación de profesores del Programa de Derecho y del Área de Filosofía de la Universidad de Ibagué, de invitados, tanto abogados como filósofos de diferentes partes del país y de estudiantes de varios programas y universidades. Se espera que este encuentro se continúe realizando, 
para seguir afianzando esta relación y permitir así que la comunidad académica se beneficie de discusiones que interesan no solo a abogados y filósofos, sino a toda la ciudadanía.

Además de los productos mencionados, como un resultado fundamental de los diálogos iniciados, actualmente existe en la Universidad de Ibagué un canal de comunicación constante entre los profesores del Programa de Derecho y el Área de Filosofía, que pueden impactar de manera muy significativa a la comunidad académica y a la formación de los futuros abogados. A continuación, se presentan unas reflexiones al respecto.

\section{El plan de estudios y los temas filosóficos}

Son muchas las relaciones existentes entre la filosofía y el derecho, así como múltiples los debates que surgen de ellas; sin embargo, se aprovecha la experiencia vivida en la Universidad de Ibagué para reflexionar sobre dos cuestiones fundamentales; en primer lugar, se trata de cómo involucrar más temas de filosofía en el plan de estudios, lo cual puede aportar a la formación de los futuros abogados y en segundo lugar, cómo trabajar con un área o programa de filosofía para que se fortalezca un programa de derecho.

En este aparte se aborda el tema de la importancia de la relación entre la filosofía y el derecho desde el punto de vista de los contenidos, específicamente, cómo incluir en el plan de estudios algunas asignaturas o temas relacionados con la filosofía, y de qué manera esto puede influir positivamente en la formación de los abogados. Para desarrollar este punto, es necesario aclarar que, para el caso del Programa de Derecho de la Universidad de Ibagué, aunque son múltiples las asignaturas relacionadas con la filosofía que se incluyeron en el nuevo plan de estudios, es posible agruparlas en cuatro grandes áreas, que pueden considerarse de amplio interés de la filosofía, que son: 1. Filosofía Política, 2. Ética, 3. Lógica y Retórica, 4. Filosofía del Derecho.

Acerca de la filosofía política, es importante destacar lo afirmado por Carlos Nino sobre la relación entre el derecho y la política:

La tesis central de esta monografía es que el derecho es un fenómeno esencialmente político, es decir, que tiene relaciones intrínsecas con la práctica política. Algunas de estas relaciones son directas, y otras se dan a través de la moral. La relación entre derecho y política parece obvia para un observador sin prejuicios teóricos, ya que todo el mundo entiende que el derecho de una sociedad varía de acuerdo con las relaciones de poder político que se dan en esa sociedad. Sin embargo, a lo largo de la historia del derecho en los países occidentales, ha habido intentos deliberados y sistemáticos de separar el derecho de la política. (Nino, 2014, p. 15).

Dada esta relación, parece claro que el estudio de la política es relevante para entender mejor el derecho y su funcionamiento. Y si esto es así, no puede negarse que es necesario introducir en los planes de estudios de derecho asignaturas que aborden la filosofía política, así como otras disciplinas que estudien los fenómenos políticos. En relación con estas disciplinas, Palacios Mejía sostiene que: 
En seguida procuraremos exponer el punto de vista que nos permite calificar como políticos algunos fenómenos sociales, y los métodos de acuerdo con los cuales es posible analizar los fenómenos políticos. Esa exposición nos permitirá definir la ciencia política, la filosofía política, el derecho constitucional y la teoría del Estado, pues todas estas disciplinas tienen en común el estudio de fenómenos políticos. (Palacios, 1980, p. 4).

Es decir que, de acuerdo a lo citado, resulta fundamental y relevante el estudio de la filosofía política, así como de todas esas disciplinas que estudian los fenómenos políticos, para que el estudiante de derecho no se limite a memorizar los textos normativos, sino que comprenda mejor las dinámicas que dan lugar a la creación y modificación del derecho. Al respecto, Palacios Mejía agrega que:

En la misma forma, y al menos desde un punto de vista teórico, podría decirse que el uso de la facultad de crear, aplicar, y definir el derecho no es ya 'política' sino 'administración, 'legislación' o 'jurisdicción'. La política es la búsqueda del control sobre las representaciones jurídicas de la comunidad. El ejercicio del poder, en cuanto pueda concebirse como separado del propósito de consolidar, extender o modificar este, no es 'política. Hay aquí, sin duda, multiplicidad de matices que ningún otro criterio permitiría evitar. (Palacios Mejía, 1980, p. 11).

Es importante destacar que dichas disciplinas políticas deben considerarse en muchas otras asignaturas, pero trabajarse con énfasis en asignaturas como Historia de las Ideas Políticas, Teoría Constitucional y del Estado y, por supuesto, en Filosofía del Derecho.

En relación con la ética, Adela Cortina (2013) sostiene que: "La ética sirve, entre otras cosas, para recordar que es una obligación ahorrar sufrimiento y gasto haciendo bien, lo que sí está en nuestras manos, como también invertir en lo que vale la pena” (p. 21).

En virtud de lo anterior, parece claro que la Ética debe ser una asignatura central en cualquier estudio, tanto en educación primaria y secundaria, como en la educación superior, mucho más cuando Adela Cortina (2013) agrega que:

Para ganar músculo ético es necesario quererlo y entrenarse, como el deportista que intenta día a día mantenerse en forma para intentar ganar limpiamente competiciones y anticiparse a los retos que estén por venir. Con eso no se solucionarán todos los problemas, pero sí que estaríamos mucho mejor preparados para buscar en serio soluciones con altura humana y para ponerlas en marcha. (Cortina, 2013, pp. 24-25).

En este mismo sentido, sobre la enseñanza de la ética en la Universidad y de esta como eje fundamental de la formación profesional, Echeverría-Falla sostiene que:

La responsabilidad social comienza con la actitud que adoptamos ante la forma de plantearnos la vida, en no ser víctimas del consumismo salvaje, en no descargar las culpas sobre el gobernante que desvió las partidas destinadas al desarrollo, en no anteponer el 'yo' al nosotros, en ser verdaderos defensores de la libertad y de la verdad. Estoy convencida de que superando estas dicotomías lograremos dar pasos consistentes para forjar en nuestros países una cultura de la solidaridad. Pasos que 
deben darse también a nivel académico, en la enseñanza universitaria, que constituyen peldaños aún por superar. Me refiero concretamente a la enseñanza de la ética como disciplina filosófica. (Echeverría-Falla, 2013, p. 154).

Así las cosas, pareciera que el estudio de la ética parece más que necesario en la educación en general y, por supuesto, la enseñanza del derecho no puede ser la excepción. No obstante, es importante tener en cuenta lo expresado por Anzola, cuando afirma que:

De esta forma, la agenda que yo persigo con mi Curso de Ética Profesional es hacer a los estudiantes realmente conscientes de que la práctica del derecho no se limita a la aplicación neutral y técnica del conocimiento jurídico, sino que, por el contrario, implica unas decisiones políticas que tienen unos impactos materiales sobre las vidas de sus clientes y de la sociedad en general; vista así, se vuelve sumamente compleja. (Anzola, 2015, p. 11).

Estas afirmaciones de Anzola, en relación a cómo desarrolla su curso de Ética Profesional, cobra relevancia en el contexto colombiano cuando hechos recientes dejan en entredicho la conducta de funcionarios de algunos sectores de la administración de justicia en el país, al poner en tela de juicio a los profesionales del derecho y con ellos a las universidades de las que han sido egresados. Al respecto, Anzola agrega que:

La ética profesional del abogado es un tema que tiene un tratamiento ambiguo por parte de la profesión legal y la academia jurídica colombiana: aunque frecuentemente manifiestan su preocupación ante la ciudadanía, principalmente a través de los medios de comunicación, sobre la poca ética que tienen algunos sectores de la profesión legal, a su interior, son escasas las medidas que se adoptan para darle a la ética profesional del abogado un lugar importante no solo dentro del currículo de los programas de pregrado, sino también en la investigación adelantada por las universidades. (Anzola, 2015, p. 4).

Ante esta situación, no debe obviarse que la reflexión ética se torna fundamental en la formación de los estudiantes de derecho.

En el nuevo plan de estudios del Programa de Derecho de la Universidad de Ibagué se incluyen las asignaturas de Ética y Política y Ética Profesional, en las que la reflexión ética debe ser el objetivo principal, aunque dicha reflexión debería hacerse de manera transversal en todas las asignaturas, porque un profesional del derecho no solo debe pensar la ética y ser ético en el aula, sino en el ejercicio de la profesión y en su vida en general.

Junto a lo anterior, y como uno de los ejes fundamentales de la formación jurídica contemporánea, se encuentra el estudio de la lógica y la retórica, áreas que por tradición han sido de interés y trabajo de los filósofos, pero que cada vez están más presentes en el estudio del derecho y pareciera que cobran mayor relevancia en un sistema de administración de justicia como el colombiano, que tiende a la oralidad.

En el Programa de Derecho, consciente de los cambios que ha tenido el sistema jurídico colombiano, se ha incorporado el estudio de estas áreas en las asignaturas de Litigación Oral de 
segundo semestre, Lógica y Retórica del tercer semestre y Argumentación Jurídica del cuarto. Aunque al igual que la ética, las competencias argumentativas deberían practicarse en casi todas las demás asignaturas.

Por último, aparece la filosofía del derecho sobre cuyo tema ha sostenido Kaufmann:

Hemos dicho que el tema de la filosofía del derecho es el 'derecho justo', la 'justicia'. Por eso sus dos preguntas básicas rezan: 1. ¿Qué es derecho justo? y 2. ¿Cómo conocemos o realizamos derecho justo? De ambas preguntas surgen conjuntamente las tareas de la filosofía del derecho: desarrollar una teoría de la justicia racional como medida de valoración para el derecho positivo, y con ello también una doctrina sobre la validez del derecho. (Kaufmann, 1999, pp. 41-42).

Y aunque pareciera casi obvia la relevancia del estudio de la filosofía del derecho para la formación jurídica y se encuentre presente en casi todos los planes de estudio de la profesión, para muchos no es muy clara su utilidad. Sobre esta cuestión Kaufmann sostiene que:

Uno tiene que encontrarse frecuentemente con la peregrina opinión según la cual ocuparse de la filosofía del derecho sólo tiene sentido si también en el futuro se puede realmente filosofar, y que además falta el tiempo necesario. Ciertamente solo se puede ejercer profesionalmente la filosofía del derecho cuando se domina el oficio filosófico. Sin embargo no todo jurista necesita ser un filósofo del derecho profesional. Pero cada jurista debe haber percibido a lo menos una vez un gusto por la filosofía del derecho, a fin de ampliar su ámbito espiritual, su 'conciencia de los problemas'. (Kaufmann, 1999, p. 29).

En el Programa de Derecho de la Universidad de Ibagué, tanto en el pénsum anterior como en el nuevo, existe la asignatura de Filosofía del Derecho, que tanto antes como ahora, tomando conciencia de la necesidad de fundar unas bases sólidas para reflexión filosófica sobre el derecho, empieza con la enseñanza de los temas fundamentales de la filosofía general, para luego conducirse hacia los temas propios del derecho.

Por todo lo anterior, se puede afirmar que la introducción de estos temas en el plan de estudios no resulta para nada irrelevante, sino todo lo contrario, no incluir asignaturas que contemplen estos temas resultaría un gran error para la formación de los abogados. Aún más si se quiere que, además de conocer los textos normativos, se adquieran competencias que permitan ejercer el derecho de manera más idónea, y esto implica no solo comprenderlo desde su relación con la política, sino ejercerlo de manera ética y con una argumentación que incluya criterios lógicos y habilidades retóricas. Junto a esto va unido por supuesto, la reflexión propia de la filosofía del derecho. 


\section{El trabajo conjunto de un área o programa de filosofía y un programa de derecho}

Es notorio que temas desarrollados por la filosofía resultan de suma relevancia para la formación jurídica, no lo es menos que para mejorar la enseñanza de estos temas y asignaturas, es necesario un trabajo y diálogo conjunto de un programa de filosofía o área de esta con un programa de derecho. Esto debido a varias razones:

La primera de ellas, es que aunque hay profesores de derecho que como abogados han estudiado y comprenden temas filosóficos, el apoyo de licenciados y filósofos a través del diálogo resulta ser de mucha utilidad para aclarar y desarrollar algunos temas, además de permitir abordar diferentes perspectivas de una situación.

En segundo lugar, en la práctica académica resulta necesario un diálogo no solo entre profesionales de la misma institución, sino con profesionales y profesores de otras instituciones, y esta relación entre un programa de derecho y un área o programa de filosofía facilita esa interacción, dados los lazos que puedan tener ambas partes con colegas de otras universidades o instituciones.

En tercer lugar y como son ejemplos, el Primer y el Segundo Encuentro de Filosofía Política y del Derecho realizados en la Universidad de Ibagué (2017-2019). El trabajo conjunto entre las áreas mencionadas permite realizar eventos con mayor amplitud, diversidad, alcance y que generen mayor impacto en la comunidad académica, pues aunque se tratan temas que tienen cierto grado de especificidad, pueden ser de mayor interés para la comunidad académica en general y no solo para los estudiantes del programa de derecho, lo cual además conlleva a la interacción con personas provenientes de otras áreas.

En cuarto lugar y como ha mostrado la experiencia del proyecto "Justicia: Un debate en torno a las cuestiones éticas contemporáneas", del cual ya se habló, las relaciones entre un programa de derecho y un programa o área de filosofía permiten un apoyo mutuo en las investigaciones, al lograr que estas adquieran un enfoque más amplio, que se ve representado en resultados de investigaciones que pueden interesar a abogados y filósofos, así como a estudiantes y profesionales de otras áreas.

\section{Conclusiones}

Aunque los aportes de la relación entre la filosofía y el derecho, en un programa de derecho se pueden evidenciar en muchísimos aspectos. De lo dicho en este artículo, se concluye que la inclusión en el plan de estudios de asignaturas que aborden temas ampliamente relacionados con la filosofía como Filosofía Política, Ética, Lógica y Retórica y Filosofía del Derecho, constituyen un gran aporte para la formación de los abogados, porque logran que estos, además de conocer las normas jurídicas, adquieran una visión más global de ellas, reconozcan la relevancia ética de su futuro actuar como profesionales, y además posean las competencias argumentativas 
que exige un sistema que tiende a la oralidad, el cual, como el derecho mismo, siempre debe ser considerado desde la reflexión propia de la filosofía del derecho.

También se puede concluir que la relación con un área o programa de filosofía, resulta beneficiosa para un programa de derecho, pues permite un diálogo entre los profesores al facilitar la reflexión sobre temas de la filosofía que le interesan al derecho y así mismo posibilita las relaciones con profesores de otras universidades, tanto de filosofía como de derecho. Esto conlleva a realizar eventos conjuntos que enriquecen las discusiones de ambas áreas y contribuye a que se desarrollen proyectos de investigación entre filósofos y abogados.

\section{Referencias}

Anzola Rodríguez, S. I. (2016). La enseñanza de "una" ética profesional del abogado a través del aprendizaje basado en problemas. Revista de Derecho Público, (37), 1-18. Recuperado de https://derechopublico.uniandes.edu.co/components/com_revista/archivos/derechopub/pub589.pdf

Cortina, A. (2013). ¿Para qué sirve realmente...? la ética. Barcelona, España: Paidós.

Echeverría-Falla, C. (2013). Educación ética: ¿Normas o virtudes? ¿Qué giro debe tomar la enseñanza de la ética en la formación de universitarios solidarios? Persona y Bioética, 17(2), 151-167. https://doi. org/10.5294/pebi.2013.17.2.1

Kaufmann, A. (1999). Filosofía del derecho. Bogotá, Colombia: Universidad Externado de Colombia.

Nino, C. S. (2014). Derecho, moral y política: Una revisión de la teoría general del Derecho. Buenos Aires, Argentina: Siglo Veintiuno Editores Argentina S. A.

Palacios Mejía, H. (1980). Introducción a la teoría del Estado. Bogotá, Colombia: Editorial Temis Ltda. 